\title{
A Relatively Short Cylindrical Broadband Antenna with Tapered Resistive Loading for Picosecond Pulse Measurements
}

\author{
MOTOHISA KANDA
}

\begin{abstract}
A relatively short cylindrical antenna with continuously tapered resistive loading has been studied for the purpose of picosecond pulse measurements. The antenna considered is a nonconducting cylinder with continuously deposited varying-conductivity resistive loading. The current distributions on the antenna were numerically calculated using the method of moments. Using these current distributions, other quantities such as input admittance, near-field and farfield radiation patterns, and radiation efficiency, were also numerically calculated and compared with the results using the Wu-King's approximate current distribution. Agreement is relatively good except at high frequencies $k h>\pi / 2$ where the method of moments appears to give better results. To verify the theoretical results, several resistively loaded antennas were fabricated, and their picosecond pulse receiving characteristics were analyzed for the frequency range between $5 \mathrm{kHz}$ and $5 \mathrm{GHz}$. The experimental results indicate excellent linear amplitude and phase response over the frequency range. This provides the unique capability of this antenna to measure fast time-varying electromagnetic fields with minimal pulse-shape distortion due to nonlinear amplitude or phase characteristics.
\end{abstract}

\section{INTRODUCTION}

$\mathbf{E}$ LECTROMAGNETIC interference (EMI) measurements are one of the most complicated and sophisticated measurements that challenge technology today since some devices and systems are much more susceptible to short-duration impulse interference than common $\mathrm{CW}$ interference. Therefore, a strong need exists to develop an isotropic broadband small sensor to detect, record, and give an alarm for impulsive electromagnetic field strengths that exceed predetermined hazardous levels. Antennas such as electrically long dipoles, pulseradiating planar arrays, long conical antennas, TEM horns, etc., have been considered previously for pulse measurements. However, these antennas become generally quite large, particularly at low frequencies. Besides, it is very difficult to make some of these antennas omnidirectional.

In $1965 \mathrm{Wu}$ and King [1] theoretically analyzed a dipole with continuous loaded complex impedance loading and predicted that the current distribution on such a dipole can be represented by an outward traveling wave if the complex impedance loading on a linear antenna is properly chosen. Shen and Wu [2] improved the theory by taking into account the step-function loading effect. More recently, a modified Fourier series solution for cylindrical transmitting antennas with tapered resistive and multiple impedance loading has been found by Taylor [3]. Following the work by Wu and King, Shen [4] and Lally and Rouch [5] have done some experimental work on relatively long (more than $25 \mathrm{~cm}$ ) resistively loaded antennas for $\mathrm{CW}$ measurements.

Manuscript received April 20, 1977; revised July 18, 1977

The author is with the EMI/Radiation Hazards Metrology Program, Electromagnetics Division, United States Department of Commerce, National Bureau of Standards, Boulder, CO 80302.
For EMI measurements, it is desirable to have a relatively short dipole (less than $10 \mathrm{~cm}$ ) which is useful for picosecond pulse (less than 100 ps duration) measurements. Therefore, the purpose of this paper is to present theoretical and experimental analyses of a relatively short broadband antenna with continuously tapered resistive loading for picosecond pulse measurements. In this paper, the current distributions on the resistively loaded antenna are calculated by solving the wave equation numerically using the method of moments. Using the current distribution obtained by the method of moments, other quantities of interest, such as input admittance, nearfield and far-field radiation patterns, and radiation efficiency, are calculated and compared with the results calculated from the Wu-King's current distribution.

To verify the theoretical results, several resistively loaded antennas were fabricated, and their picosecond pulse receiving characteristics were examined experimentally. The picosecond pulse receiving characteristics of the resistively loaded antenna were analyzed using a time domain network analyzer with 70 ps duration impulses.

\section{THEORETICAL CONSIDERATION}

The antenna consists of a hollow cylinder of length $2 h$ and radius $a$ aligned along the $z$-axis and centered at the origin. It has an internal impedance of $Z^{i}(z)$ due to continuous complex impedance loading and carries an axial current of $I_{z}(z)$, which is assumed uniform around the periphery of the cylinder since the radius $a$ is much less than the wavelength $\lambda$. When the antenna is driven by a delta function voltage $V_{0}$ at $z=0$, the axial component of the vector potential, $A_{z}(z)$, on the surface of the antenna satisfies the one-dimensional wave equation

$$
\left(\frac{\partial^{2}}{\partial z^{2}}+k^{2}\right) A_{z}(z)=\frac{j k^{2}}{\omega}\left[Z^{i}(z) I_{z}(z)-V_{0} \delta(z)\right]
$$

where

$$
A_{z}(z)=\frac{\mu_{0}}{4 \pi} \int_{-h}^{h} I_{z}\left(z^{\prime}\right) K\left(z, z^{\prime}\right) d z^{\prime}
$$

and

$$
K\left(z, z^{\prime}\right) \cong \frac{e^{-j k \sqrt{\left(z-z^{\prime}\right)^{2}+a^{2}}}}{\sqrt{\left(z-z^{\prime}\right)^{2}+a^{2}}} .
$$

When the cylindrical antenna has a continuous complex impedance loading such that the internal impedance per unit length $Z^{i}(z)$ is a particular function of the position along the antenna, $\mathrm{Wu}$ and King [1] found that a pure outward traveling 
wave can exist on an antenna of finite length. That is, if

$$
Z^{i}(z)=\frac{60 \psi}{h-|z|},
$$

where

$$
\begin{aligned}
\psi= & \frac{\int_{-h}^{h} I\left(z^{\prime}\right) K\left(z, z^{\prime}\right) d z^{\prime}}{I(z)} \\
\cong & 2\left(\sinh ^{-1} \frac{h}{a}-C(2 k a, 2 k h)-j S(2 k a, 2 k h)\right) \\
& +\frac{j}{k h}\left(1-e^{-j 2 k h}\right) \quad \text { at } z=0,
\end{aligned}
$$

and $C(a, x)$ and $S(a, x)$ are the generalized cosine and sine integrals, then the approximate current distribution for the one-dimensional wave equation (1) is of a traveling wave in nature,

$$
I_{z}(z)=\frac{V_{0}}{60 \psi(1-j / k h)}\left(1-\frac{|z|}{h}\right) e^{-j k|z|},
$$

and the solution of $e^{+j k|z|}$ does not satisfy the original wave equation. Since the frequency dependence of $Z^{i}(z)$ appears only in $\psi$ in the form of a logarithm for small $k h$, the antenna has much broader frequency characteristics than an antenna with a lumped resistor located at quarter-wavelengths from the ends [6], [7].

It should be noted, however, that the loading given by (4) is a complex impedance, since the constant $\psi$ is a complex number. As will be shown later, the real part of $\psi$ is independent of frequency, and the imaginary part of $\psi$ is negative and proportional to frequency. For this reason, it is not possible to realize the loading given by (4) using passive elements.

In the present study, a relatively short cylindrical antenna with continuously tapered resistive loading has been studied for the purpose of picosecond pulse measurements. The current distribution on the resistively loaded antenna for different frequencies is calculated by solving the one-dimensional wave equation given in (1) numerically using the method of moments [8]. To simplify the integral equation, it is assumed that a wire radius $a$ is much smaller than a wavelength $\lambda, a \ll \lambda$, so that the current can be considered to flow in a filament along the $z$-axis rather than on the surface of a cylinder. To permit a solution for the current distribution, suitable testing functions and basis functions for expansion are defined. Here we choose piecewise linear testing functions and employ piecewise constant basis functions for expansion to solve for the current distribution on the resistively loaded antenna. Other choices of testing functions and basis functions for expansion are, of course, possible, e.g., piecewise constant, piecewise linear, piecewise sinusoidal, etc. Combinations other than piecewise linear testing functions and piecewise constant basis functions for expansion of the current might lead to better convergence. It is beyond the scope of this paper to discuss the convergence of series representations using a different combination of testing functions and basis functions for expansion. A detailed discussion on the subject is given by Butler and Wilton [9] .
Applying the moment method technique to the one-dimensional wave equation, the set of the wave equation may be written in matrix form as [10]

$$
[V]=[Z][I]
$$

where

$$
\begin{aligned}
Z_{m, n}= & j \frac{\omega}{k^{2}} \frac{1}{\Delta} \frac{\mu_{0}}{4 \pi} \int_{\xi_{n}-\frac{\Delta}{2}}^{\xi_{n}+\frac{\Delta}{2}}\left(K\left(z_{m+1}, z^{\prime}\right)\right. \\
& \left.-2\left(1-\frac{(k \Delta)^{2}}{2}\right) K\left(z_{m}, z^{\prime}\right)+K\left(z_{m-1}, z^{\prime}\right)\right) d z^{\prime} \\
& +\Delta \cdot Z^{m}\left(z_{m}\right) P_{n}\left(z_{m}\right)
\end{aligned}
$$

and the $P_{n}\left(z_{m}\right)$ are the basis functions used for the expansion of current, i.e., piecewise constant in this case, and $\Delta=z_{m}-$ $z_{m-1}$. [ $\left.V\right]$ is the voltage excitation matrix. The elements of the matrix $[I]$, which give the current distribution, can then be obtained by solving the matrix equation ( 7 ).

The numerical solutions using the method of moments and the analysis based on the approximations by $\mathrm{Wu}$ and King have been applied to the resistively loaded antenna. In the method of moments, the current distributions are calculated by subdividing the dipole into 61 subsections. In order to observe the convergence of the series representation for current on the dipole, a numerical solution with 121 subsections was also examined. The results indicate that the convergence is very good for a numerical solution with 61 subsections.

\section{FABRICATION OF CONTINUOUSLY TAPERED RESISTIVELY LOADED ANTENNAS}

The resistive antenna elements are made by depositing a linearly tapered thin-film alloy on a glass rod. This alloy consists of 74.50 percent nickel, 20 percent chromium, 2.75 percent aluminum, and 2.75 percent copper, and has a relatively high resistivity of $133 \mu \Omega-\mathrm{cm}$ and a very low temperature coefficient of $\pm 0.00002 /^{\circ} \mathrm{C}$. A glass rod, $7.5 \mathrm{~cm}$ long and $0.254 \mathrm{~cm}$ in diameter, was chosen as a substrate for the deposited element. This becomes a quarter-wave monopole, $k h=\pi / 2$, at $1 \mathrm{GHz}$. For a dipole configuration, a $15 \mathrm{~cm}$ long glass rod is used as a substrate for the deposited element. Once the antenna dimensions are determined, the desired resistive loading profile is calculated using (4). The desired resistive loading profile, which turns out to be a relatively weak function of frequency, is calculated at $1 \mathrm{GHz}$ and is shown in Fig. 1. Here, the constant $\psi$ in (5), the ratio of the vector potential to the current along the antenna, which is in general complex, is assumed to be real by taking the absolute value of the complex constant $\psi$. Typically, the resistive loading required is about $5 \mathrm{k} \Omega / \mathrm{m}$ at the driving point, about $10 \mathrm{k} \Omega / \mathrm{m}$ at the midpoint, and infinite at the end of the antenna. Knowing the resistivity of the alloy used, i.e., $133 \mu \Omega-\mathrm{cm}$, it is possible to calculate the required thin-film thickness of the alloy, which is shown in Fig. 2. Typically, the thickness of the thin-film alloy is about $24 \mathrm{~nm}$ at the driving point, $10 \mathrm{~nm}$ at the midpoint, and zero at the end of the antenna.

The $15 \mathrm{~cm}$ long glass rod was placed in the bell jar tilted at an angle. The tilt angle is adjusted to obtain the proper resis- 


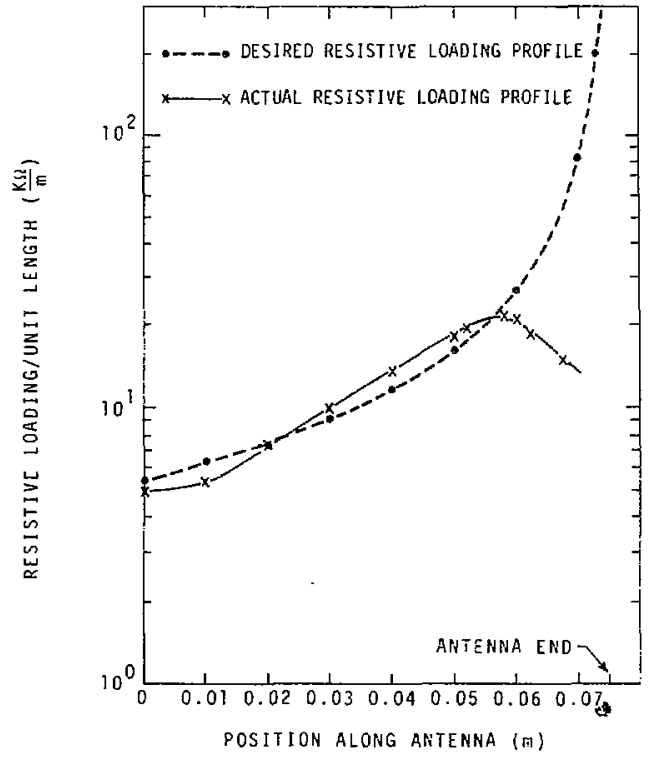

Fig. 1. Resistive loading profile.

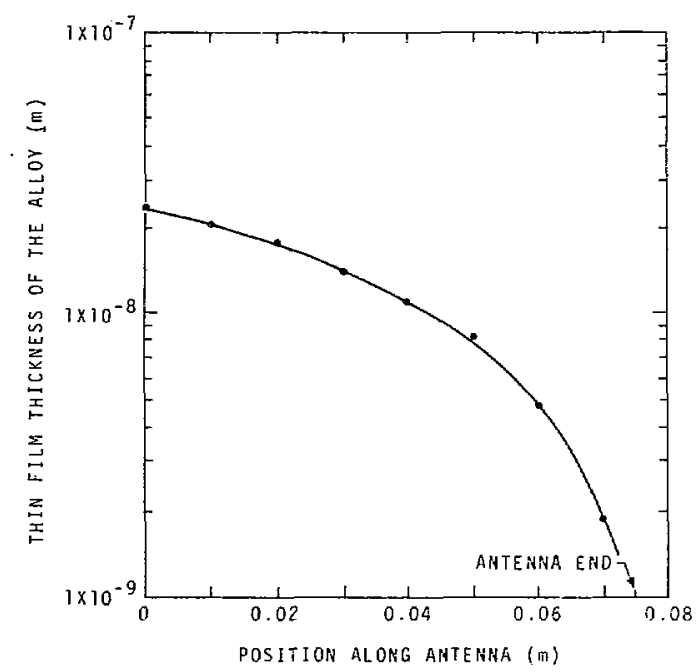

Fig. 2. Required profile of thin-film thickness of alloy.

tive loading profile. The line source of the depositing alloy is used so that the resistive loading profile has a dependence of inverse distance, rather than a dependence of inverse distance squared for the point source of a depositing alloy. The photograph of the resistively loaded monopole antenna is shown in Fig. 3.

\section{COMPARISON BETWEEN MOMENT METHOD SOLUTIONS AND WU-KING APPROXIMATE SOLUTIONS}

First, the current distributions on the resistively loaded antenna were calculated using both the method of moments and the Wu-King approximation. These results were compared with the experimental results. Once the current distributions were found, all other antenna characteristics, such as input admittance, near-field and far-field radiation patterns, and radiation efficiency, were then calculated. In this section, these antenna characteristics are calculated from the current distributions obtained by both the method of moments and the $\mathrm{Wu}$ -

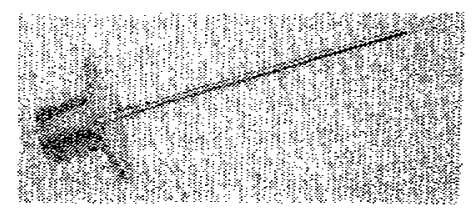

Fig. 3. Photograph of resistively loaded monopole.

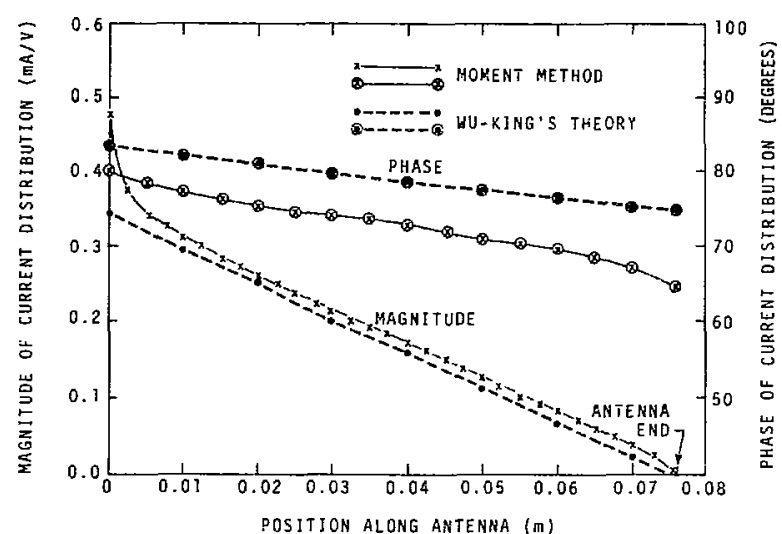

(a)

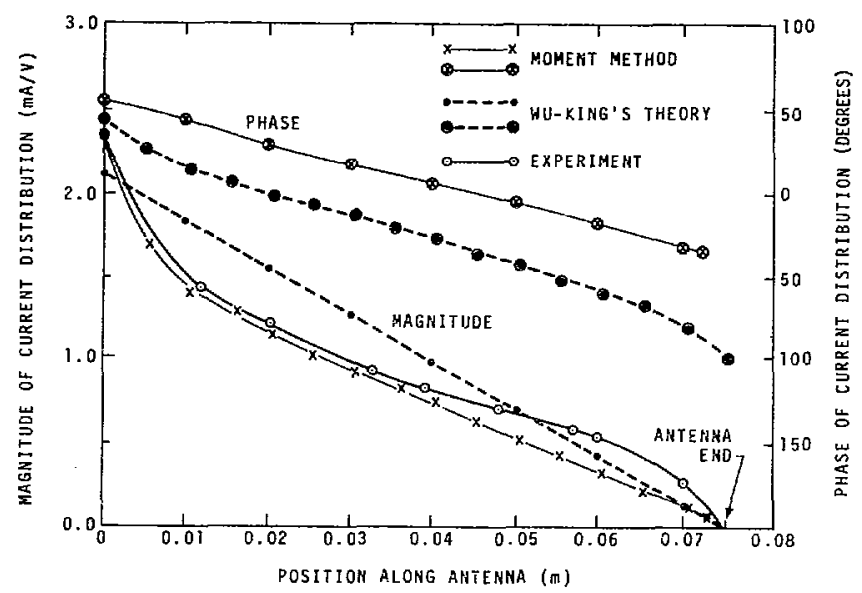

(b)

Fig. 4. Current distribution on resistively loaded antenna. (a) At $100 \mathrm{MHz}$. (b) At $1 \mathrm{GHz}$.

King approximation, and are, in turn, compared with the experiments.

\section{A. Current Distribution}

The magnitude and the phase of the current distributions on the resistively loaded antenna are calculated using both the method of moments and the Wu-King approximation, and are shown in Fig. 4(a) and (b) for different frequencies. The experimental results of the current distribution at $1 \mathrm{GHz}$ are also shown in Fig. 4(b). The current distribution measurements were made using a $1 \mathrm{~cm}$ diameter loop with a beam lead Schottky diode as shown in Fig. 5. High-resistance $(40 \mathrm{M} \Omega / \mathrm{m})$ lines were used in order to connect to the loop to minimize distortion of both the field and the interaction with the loop antenna. The combination of resistors and capacitors forms a low pass filter to prevent RF interaction between the loop antenna and the dc measurement devices. Generally, at frequencies below $1 \mathrm{GHz}, k h<\pi / 2$, the agreement between the moment method solutions, the Wu-King approximate solutions, and the experimental results is extremely good. However at 


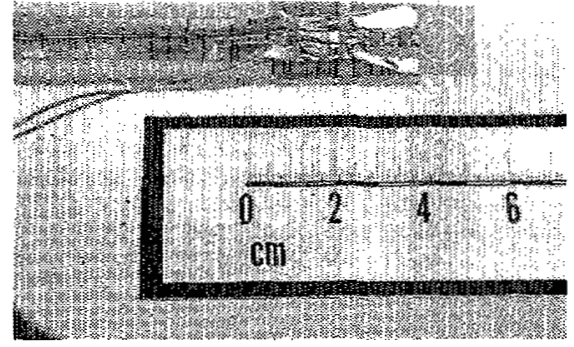

Fig. 5. Photograph of loop antenna used for current distribution measurements.

frequencies above $1 \mathrm{GHz}, k h \geq \pi / 2$, the discrepancies between them are great, and the experimental results on current distributions agree well with the solutions obtained by the method of moments.

\section{B. Driving Point Admittance}

The driving point admittance using the current distribution was obtained by both the method of moments and the WuKing approximation, and is compared with the experimental results. The driving conductance and the driving susceptance of the antenna are, respectively, shown in Fig. 6(a) and (b). Using the Wu-King approximation, the driving point admittance is given by

$$
Y_{0}=\frac{1}{60 \psi} \frac{1}{1-j / k h}
$$

The experimental results were obtained by using an impedance bridge for the frequency range from $10 \mathrm{MHz}$ to $300 \mathrm{MHz}$ and an automatic network analyzer for the frequency range from $100 \mathrm{MHz}$ to $18 \mathrm{GHz}$. The agreement between theory and experiments is relatively good except at frequencies above $1 \mathrm{GHz}, k h>\pi / 2$. The resistively loaded monopole was mounted in a type $N$ connector mount. The discrepancy between theory and experiments at frequencies above $1 \mathrm{GHz}$, $k h>\pi / 2$ is due mainly to the fact that the theory neglects the effect of the type $N$ connector mount which typically has a capacitance of about $1 \mathrm{pF}$. This extra capacitance caused an increase in antenna susceptance as shown in Fig. 6(b). At frequencies below $1 \mathrm{GHz}, k h<\pi / 2$, the impedance of the resistively loaded antenna is a resistance of $240 \Omega$ and a capacitance of $0.08 \mathrm{pF}$ in series configuration.

\section{C. $\psi$}

The ratios $\psi$ of vector potential to current at the antenna driving point are calculated by using both the current distributions from the method of moments and the Wu-King approximation. The real and imaginary parts of $\psi$ are, respectively, shown in Fig. 7(a) and (b). At low frequencies below $1 \mathrm{GHz}$, $k h<\pi / 2$, the agreement is very good between the ratio $\psi$ calculated from the current distributions by the method of moments and that calculated from the current distributions by the Wu-King approximation. At high frequencies above $1 \mathrm{GHz}$, $k h>\pi / 2$, the discrepancy is large, mainly due to the fact that the current distributions by the Wu-King approximation are not sufficiently accurate.

It is found from Fig. 7(a) and (b) that the ratio $\psi$ of vector potential to current at the antenna driving point is a very slowly varying function of frequency, since the frequency

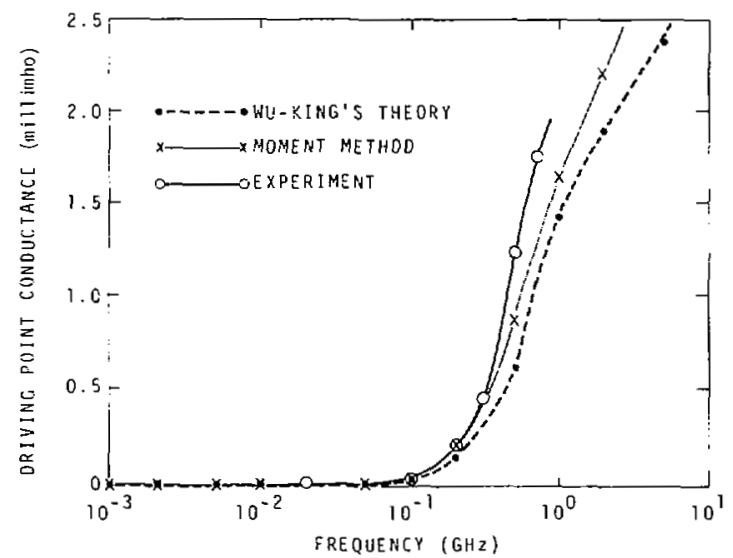

(a)

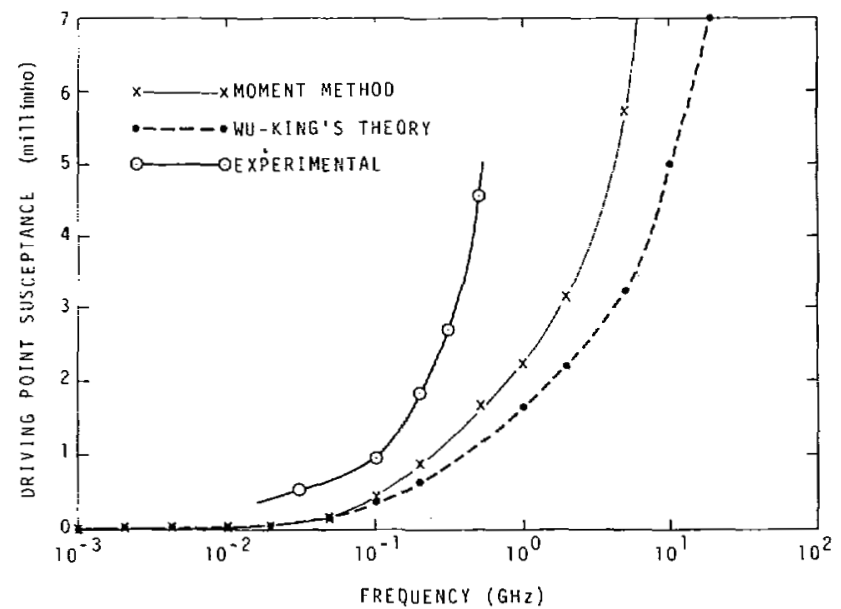

(b)

Fig. 6. Driving point admit tance of resistively loaded dipole. (a) Driving point conductance. (b) Driving point susceptance.

dependence of $\psi$ in the approximate formula given in (5) appears only in the form of a logarithm. Therefore, the resistive loading profile $Z^{i}(z)$ given in (4) and the current distribution $I_{z}(z)$ given in (6) have a very weak frequency dependence, which results in the broad-band characteristics.

\section{Near-Field and Far-Field Radiation Patterns}

The electric field of the resistively loaded antenna for both the near-field and the far-field regions is rigorously given by

$$
\begin{aligned}
E_{\theta}(r) & =j \omega \sin \theta A_{z} \\
& =\frac{j \omega \mu_{0} \sin \dot{\theta}}{4 \pi} \int_{-h}^{h} I_{z}\left(z^{\prime}\right) \frac{e^{-j k \sqrt{r^{2}+z^{\prime 2}+2 r z^{\prime} \cos \theta}}}{\sqrt{r^{2}+z^{\prime 2}+2 r z^{\prime} \cos \theta}} d z^{\prime} .
\end{aligned}
$$

This integration is carried out numerically using the current distribution $I_{z}(z)$ obtained by the method of moments.

Using the current distribution given by the Wu-King approximation, the electric fields for both the near-field and the far-field regions are also calculated using (10) with the current distribution given in (6). In the far-field region, the electric field can be calculated analy tically [1], yielding

$$
E_{\theta} f(r)=\frac{j V_{0}}{\psi(1-j / k h)} \cdot \frac{F(k h, \theta)}{r}
$$




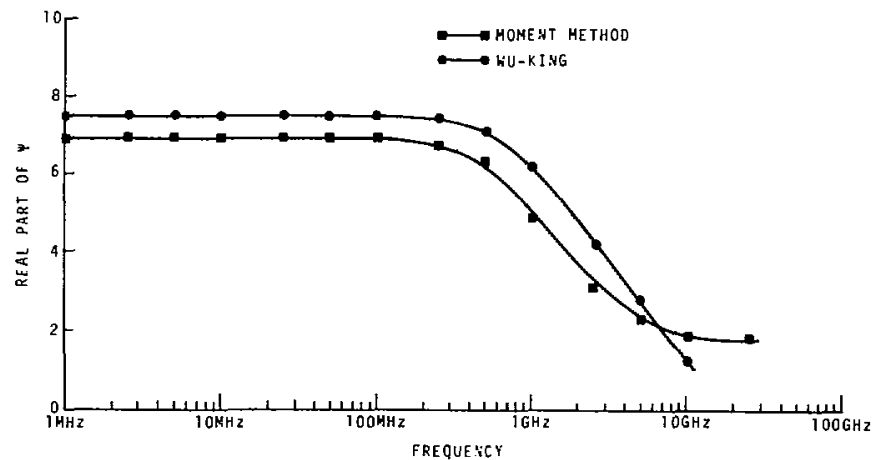

(a)

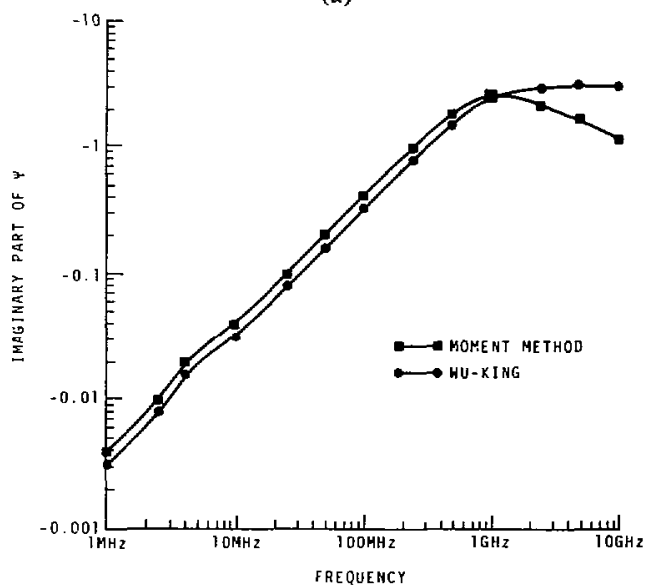

(b)

Fig. 7. $\psi$ as a function of frequency. (a) Real part of $\psi$. (b) Imaginary part of $\psi$.
$\mathrm{GHz}, k h=10 \pi / 2$, is shown in Fig. 8(e). It was thus found that the $15 \mathrm{~cm}$ long resistively loaded dipole discloses a wellbehaved electric field pattern up to $3 \mathrm{GHz}, k h=3 \pi / 2$, whereas a $15 \mathrm{~cm}$ long metal dipole has a well-behaved electric field pattern up to $1 \mathrm{GHz}, k h=\pi / 2$, as expected.

The near-field radiation patterns are also calculated numerically for both the resistively loaded dipole and a metal dipole. The theoretical results are shown in Fig. 9 for several frequencies. The near-field radiation patterns are calculated and measured at $10 \mathrm{~cm}$ away from the dipole. In the measurements, a broadband balun or $3 \mathrm{~dB}$ hybrid coupler is used to match the balanced dipole to a $50 \mathrm{ohm}$ unbalanced $\mathrm{CW}$ generator. A very short dipole with a Schottky barrier beam lead diode was used to probe near-field radiation patterns. At frequencies below $1 \mathrm{GHz}, k h<\pi / 2$, near-field radiation patterns as shown in Fig. 9(a) and (b) for both the resistively loaded dipole and a metal dipole are very similar to far-field radiation patterns. However, at frequencies above $1 \mathrm{GHz}, k h>\pi / 2$, the near-field radiation pattern of the resistive loaded antenna splits and results in sidelobes as shown in Fig. 9(c). The agreement between theory and experiments is quite good as indicated in Fig. 9(b).

\section{E. Radiation Efficiency}

An obvious concern for a resistively loaded antenna is the reduced radiation efficiency of such antennas. The radiation efficiency is defined as the ratio of the total power radiated by an antenna to the net power accepted by the antenna [12]. The radiated power $P_{r}$ is calculated by integrating the Poynting vector over a sphere and is given by

where

$$
F(k h, \theta)=\frac{-j k h \sin ^{2} \theta+\left(1+\cos ^{2} \theta\right)-\left[j 2 \cos \theta \sin (k h \cos \theta)+\left(1+\cos ^{2} \theta\right) \cos (k h \cos \theta)\right] e^{-j k h}}{k h \sin ^{3} \theta} .
$$

For comparison, the near-field and the far-field radiation patterns for a metal dipole are also calculated using the method of moments.

The experiments to obtain the field radiation pattern were performed using a near-field calibration range [11] for the frequency range between $700 \mathrm{MHz}$ and $5 \mathrm{GHz}$. A beam lead Schottky diode was mounted at the dipole driving point and was connected to a high common-mode rejection, highimpedance amplifier by means of high resistance $(50 \mathrm{k} \Omega / \mathrm{m})$ lines in order to minimize both distortion of the field and interaction with the dipole.

The experimental results of the far-field radiation patterns are shown in Fig. 8 for several frequencies along with the theoretical results. As shown in Fig. 8 (a) and (b), at frequencies below $1 \mathrm{GHz}, k h<\pi / 2$, the electric field patterns of the resistively loaded antenna are well-behaved and are very similar to those of a metal dipole with the same electrical length, $h=$ $7.5 \mathrm{~cm}$. As a frequency increases above $1 \mathrm{GHz}, k h>\pi / 2$, the electric field patterns of a $15 \mathrm{~cm}$ long metal dipole split and result in many sidelobes as shown in Fig. 8(c)-(e). However, Fig. $8(\mathrm{c})$ indicates that the $15 \mathrm{~cm}$ long resistively loaded dipole, $k h=\pi / 2$ at $1 \mathrm{GHz}$, has a well-behaved electric field pattern up to $3 \mathrm{GHz}, k h=3 \pi / 2$. At $5 \mathrm{GHz}, k h=5 \pi / 2$, the electric field pattern of the resistively loaded dipole splits and results in sidelobes as shown in Fig. 8(d). For further reference, the electric field pattern of the resistively loaded antenna at 10

$$
P_{r}=\frac{1}{2 \zeta_{0}} \int_{0}^{2 \pi} d \phi \int_{0}^{\pi} \sin \theta \cdot|E(\theta, \phi)|^{2} \cdot r^{2} d \theta
$$

where $\zeta_{0}$ is a free-space impedance. The dissipated power due to resistive loading is calculated by

$$
P_{h}=2 \int_{0}^{h} \frac{1}{2}|I(z)|^{2} Z^{i}(z) d z
$$

Thus the radiation efficiency is given by

$$
\eta_{r}=\frac{P_{r}}{P_{h}+P_{r}}
$$

The current distribution on the resistively loaded antenna obtained by the method of moments is used to numerically calculate the radiation efficiency, and the result is shown in Fig. 10.

In the far-field range, using the approximate current distribution given by $(6)$, the radiated power, $P_{r}$, is given by [1]

$$
P_{r}=\frac{2 \pi V_{0}^{2}}{\zeta_{0}|\psi|^{2}\left(1+\frac{1}{k^{2} h^{2}}\right)} \int_{0}^{1} F^{2} d y
$$




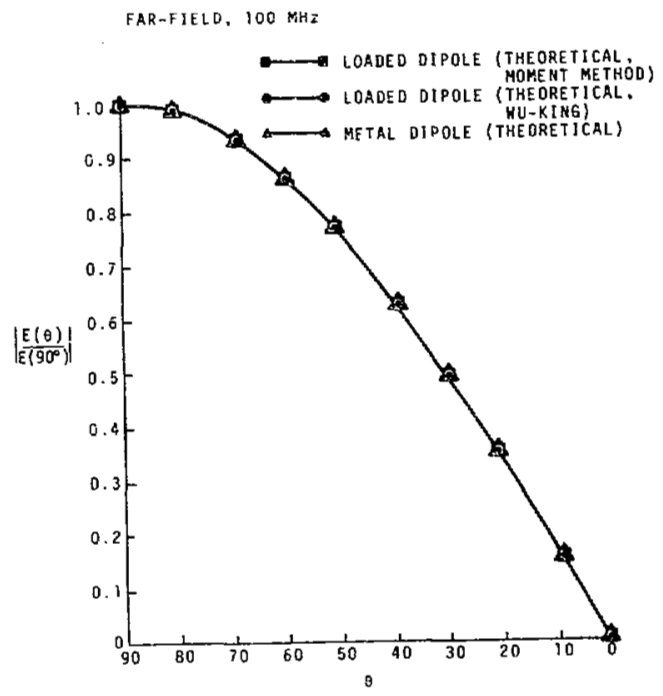

(a)

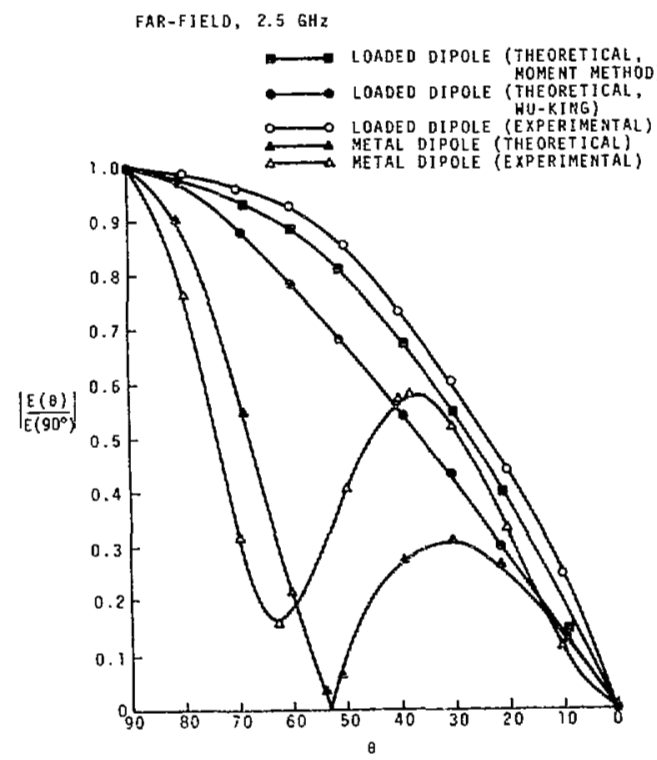

(c)

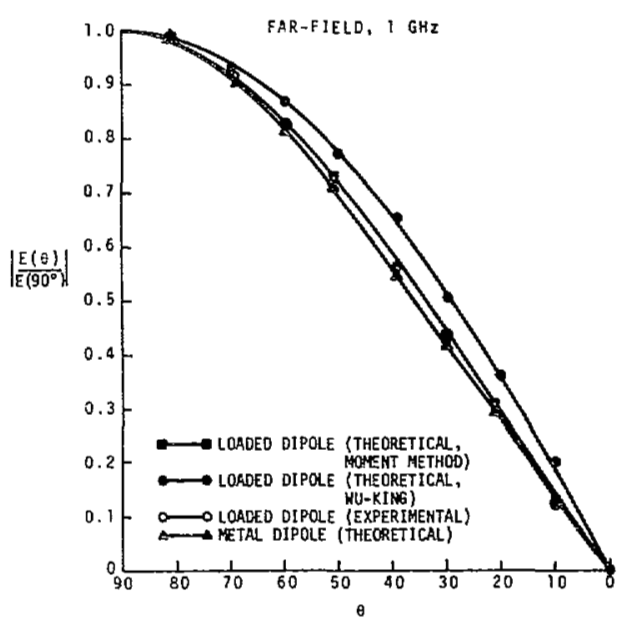

(b)

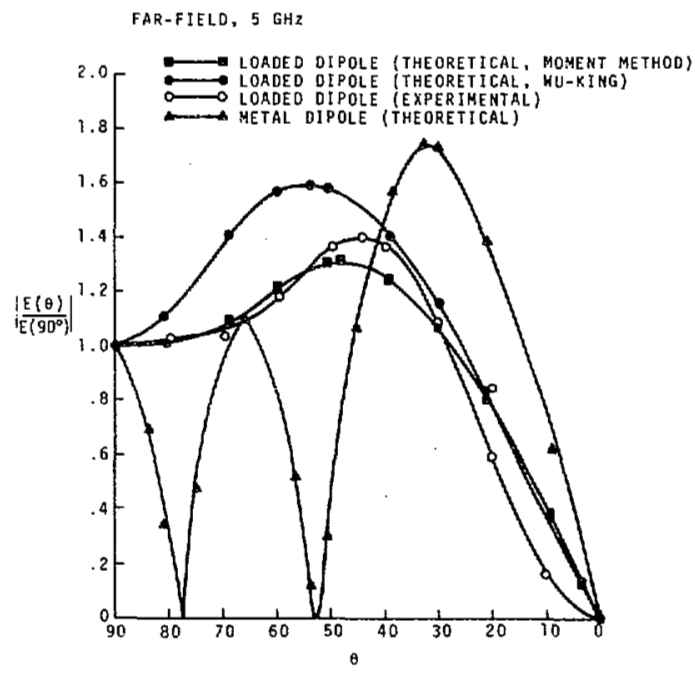

(d)

FAR-FIELD, $10 \mathrm{GHZ}$

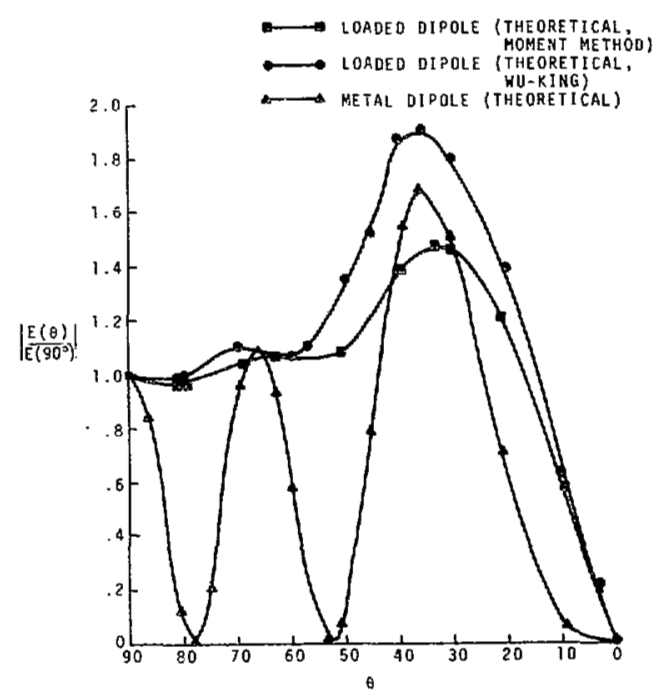

(e)

Fig. 8. Far-field radiation patterns. (a) At $100 \mathrm{MHz}$. (b) At $1 \mathrm{GHz}$. (c) At $2.5 \mathrm{GHz}$. (d) At $5 \mathrm{GHz}$. (e) At $10 \mathrm{GHz}$. 


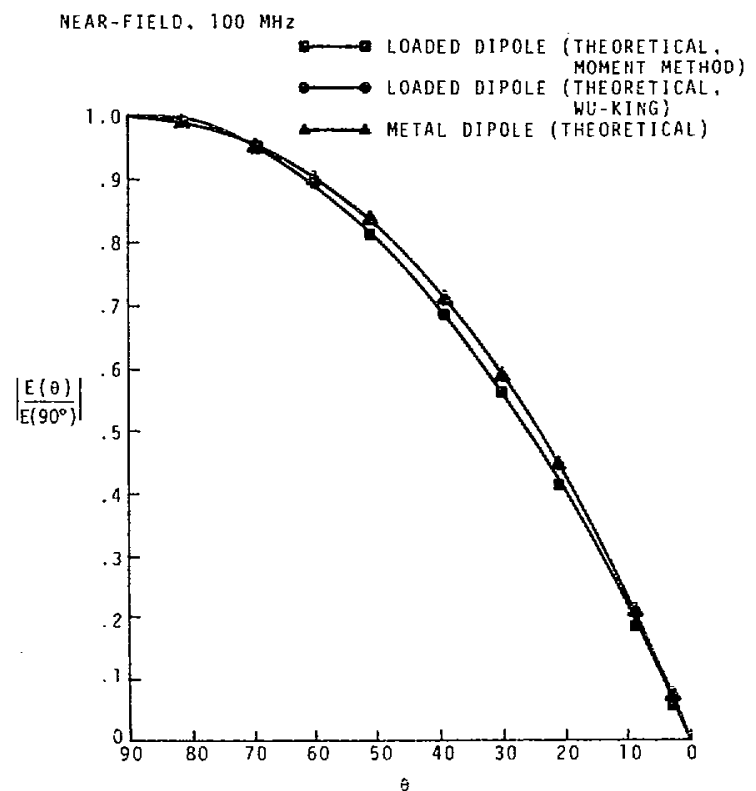

(a)

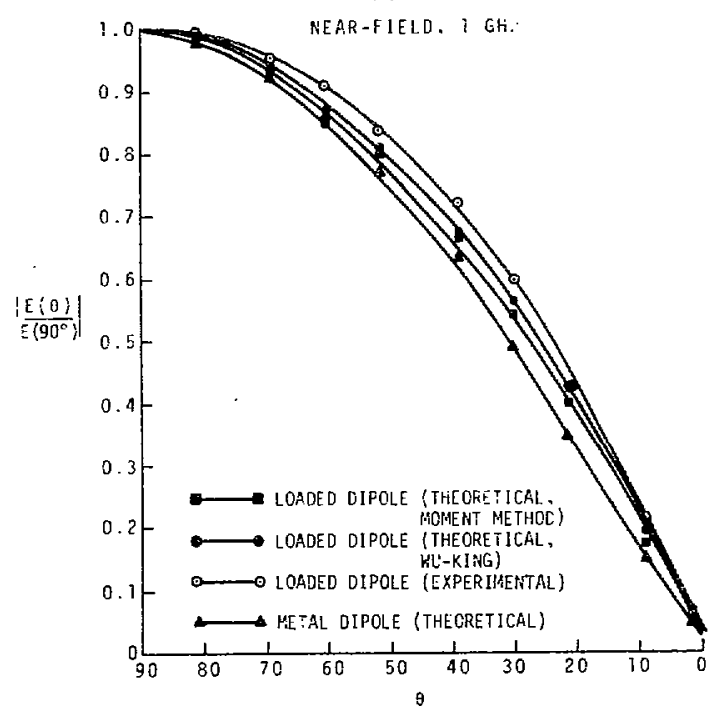

(b)

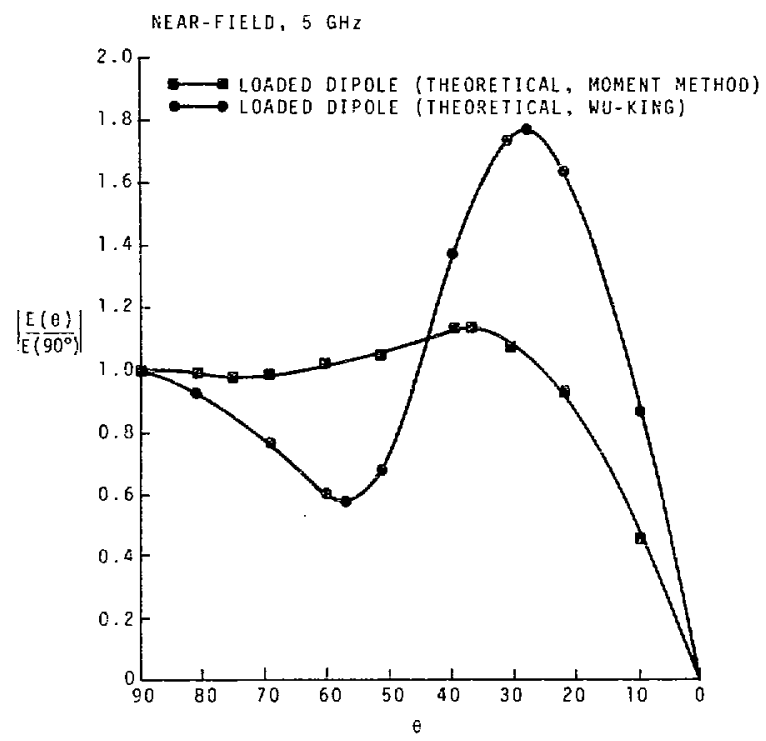

(c)

Fig. 9. Near-field radiation pattems. (a) At $100 \mathrm{MHz}$. (b) At $1 \mathrm{GHz}$. (c) At $5 \mathrm{GHz}$.

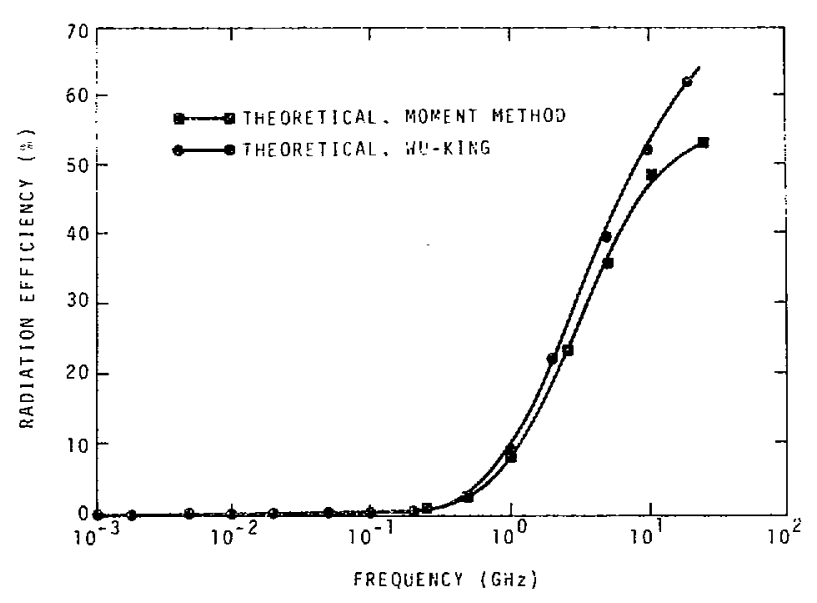

Fig. 10. Radiation efficiency of resistively loaded antenna.

where $F$ is given in (12) and $y=\cos \theta$. Under the same assumption, the dissipated power $P_{h}$ is also given as

$$
P_{h}=\frac{\pi V_{0}^{2}}{\zeta_{0}|\psi|\left(1+\frac{1}{k^{2} h^{2}}\right)}
$$

The resultant radiation efficiency is also shown in Fig. 10. The agreement between the radiation efficiency calculated by the current distribution obtained by the method of moments and that obtained from the Wu-King current distribution is relatively good except at frequencies above $1 \mathrm{GHz}, k h>\pi / 2$. At frequencies above $1 \mathrm{GHz}, k h>\pi / 2$, the experimental results on the current distribution on the resistively loaded antenna agree well with the theoretical results obtained by the method of moments. Thus, it is expected that the radiation efficiency based on the current distribution by the method of moments will give a correct value at frequencies above $1 \mathrm{GHz}, k h>\pi / 2$.

\section{マ. TIME DOMAIN MEASUREMENTS FOR THE RESISTIVELY LOADED ANTENNA WITH A $50 \Omega$ LOAD}

The transients of the resistively loaded antenna were evaluated on the time domain antenna range with a time domain automatic network analyzer (TDANA) developed by the National Bureau of Standards (NBS). The TDANA measures the time domain waveform of impulse response of the resistively loaded antenna. The time domain waveform is then digitized and stored in the minicomputer memory. By acquiring an ensemble of many waveforms, it is possible to perform signal averaging within the minicomputer to improve the signal to noise ratio in the measurements. The minicomputer then computes the spectrum amplitude from the digitized, averaged waveform using the fast Fourier transform (FFT).

The bottom curve of Fig. 11(a) shows the time domain waveform of the impulse from the impulse generator. The duration of the impulse is typically $70 \mathrm{ps}$, and its amplitude is about 5 to $6 \mathrm{~V}$. This impulse is transmitted through a $2.8 \mathrm{~m}$ long conical standard antenna and received on the resistively loaded antenna. The antenna feed points on the ground plane are separated by $1 \mathrm{~m}$. The upper curve of Fig. 11(a) shows the time domain waveform of the impulse received by the resistively loaded antenna. It should be noted how well the resis- 


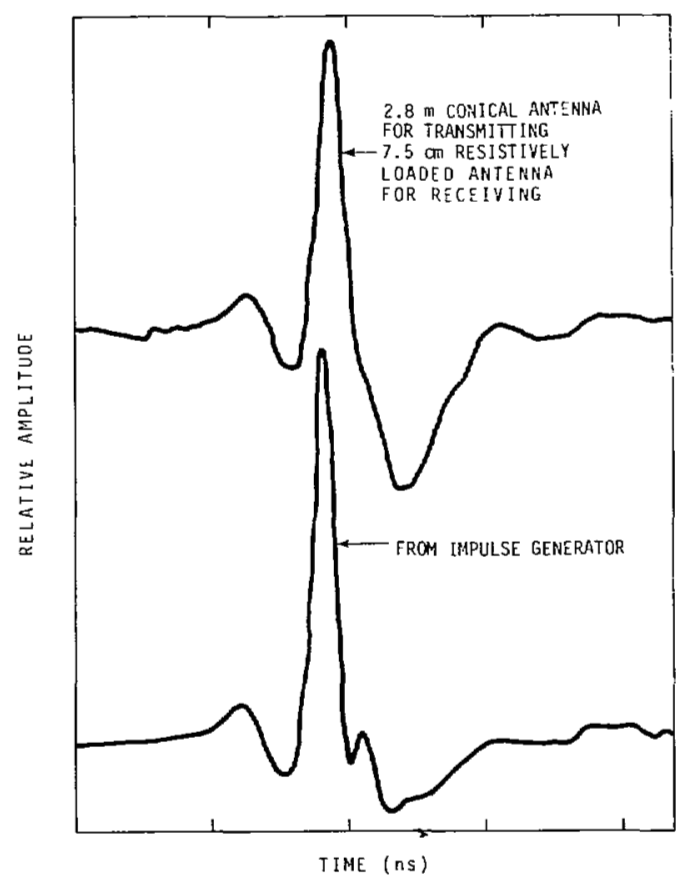

(a)

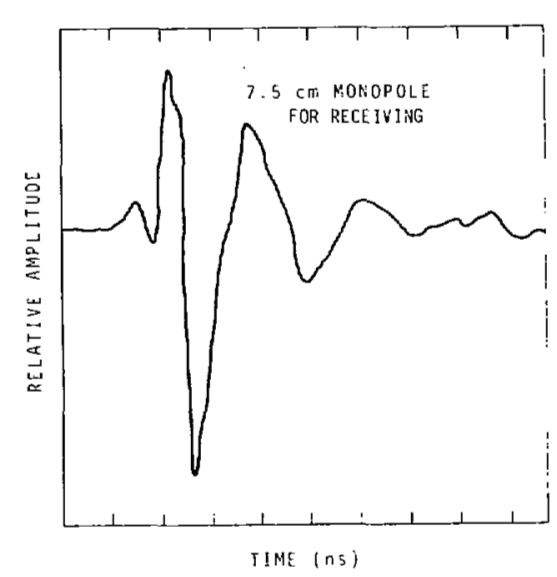

(b)

Fig. 11. Time domain impulse responses. (a) Resistively loaded monopole. (b) Metal monopole.

tively loaded antenna can preserve the waveform of the radiated impulse field from the conical standard antenna. On the other hand, when a metal dipole with the same electrical length is used for receiving the same impulse, the shape of the 70 ps impulse is completely destroyed as shown in Fig. 11 (b).

Using the time domain wave of the impulse from the impulse generator which is shown in the bottom curve in Fig. 11(a), the spectrum amplitude of the impulse is calculated by the FFT and is shown in Fig. 12. The spectrum amplitude is relatively flat with a nominal level of $60 \mathrm{~dB} \mu \mathrm{V} / \mathrm{MHz}$ to $5 \mathrm{GHz}$. Beyond $6 \mathrm{GHz}$, the spectrum amplitude drops very rapidly.

Fig. 13 shows the transfer functions of a two-antenna system, a conical standard antenna for transmitting and the 7.5 $\mathrm{cm}$ long resistively loaded monopole for receiving. It is found from Fig. 13 that the resistively loaded monopole has a frequency response that is flat to $\pm 3 \mathrm{~dB}$ from $200 \mathrm{MHz}$ to over $5 \mathrm{GHz}$.

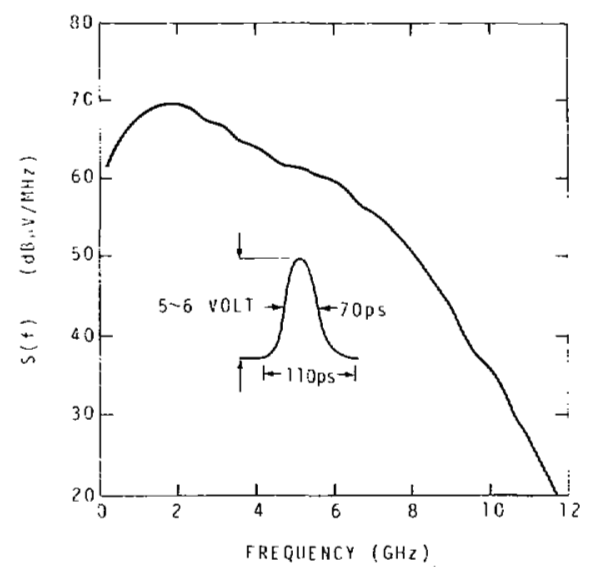

Fig. 12. Spectrum amplitude of impulse.

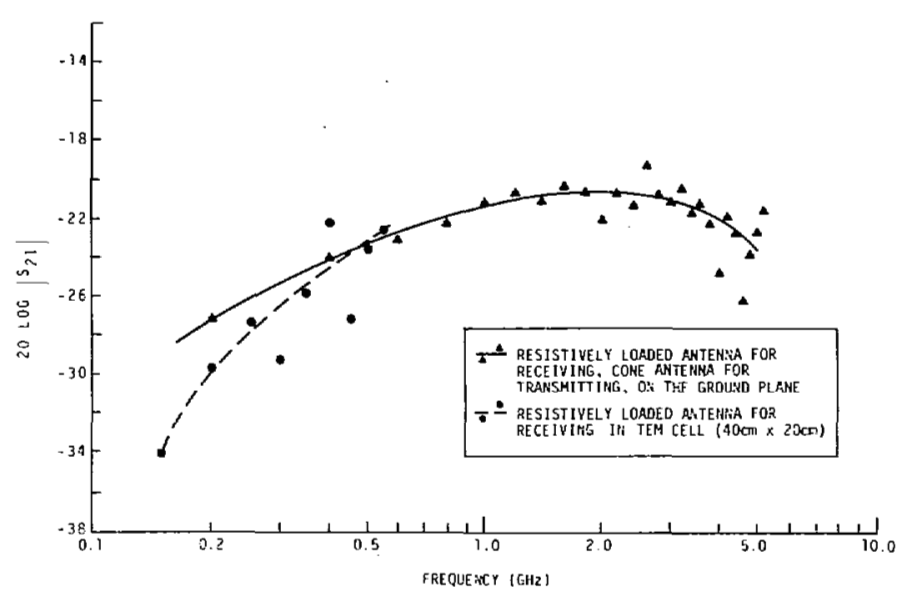

Fig. 13. Transfer function of two antennas: conical standard antenna for transmitting and resistively loaded monopole for receiving.

\section{CONCLUSION}

The characteristics of a relatively short cylindrical broadband antenna with continuous resistive loading were studied theoretically and experimentally for picosecond pulse measurements. The antenna considered is a nonconducting cylinder with continuously deposited tapered resistive loading. The current distribution on the resistively loaded antenna is calculated using the method of moments and compared with the Wu-King approximate formula. Experimental results of the current distribution on the resistively loaded antenna agree with the results of the method of moments. Using the current distributions obtained both by the method of moments and the Wu-King approximation, other quantities, such as input admittance, near-field and far-field radiation patterns, and radiation efficiency, were calculated and also examined experimentally. These experimental results agree well with the predicted solutions of both methods up to $1 \mathrm{GHz}$, but agreement is better with the moment method solutions above $1 \mathrm{GHz}$.

Time domain measurements were performed using a timedomain antenna range with a time domain automatic network analyzer. The receiving characteristics of the resistively loaded antenna indicate that the radiated impulse shape of 70 ps duration is well preserved. The resistively loaded antenna has a receiving response flat to $\pm 3 \mathrm{~dB}$ from $200 \mathrm{MHz}$ to over $5 \mathrm{GHz}$. 
The theoretical and experimental results indicate that the resistively loaded antenna processes excellent linear amplitude and phase response over the broad frequency range. This provides the unique capability of the antenna to measure fast time-varying electromagnetic fields with minimal pulse-shape distortion due to nonlinear amplitude or phase characteristics.

\section{ACKNOWLEDGMENT}

The author wishes to thank Dr. P. F. Wacker for many stimulating discussions and B. K. Bender for computer assistance. Acknowledgment should also be extended to D. G. Melquist, A. R. Ondrejka, and T. H. Bremer for their experimental assistance, and C. K. S. Miller, F. X. Ries, and J. W. Adams for many helpful discussions and encouragement during the course of this work.

\section{REFERENCES}

[1] T. T. Wu and R. W. P. King, "The cylindrical antenna with nonreflecting resistive loading," IEEE Trans. Antennas Propagat., vol. AP-13, no. 3, pp. 369-373, May 1965. This paper was corrected by L. C. Shen and R. W. P. King, "The cylindrical antenna with nonreflecting resistive loading," IEEE Trans. Antennas Propagat., vol. AP-13, no. 6, p. 998, Nov. 1965.

[2] L. C. Shen and T. T. Wu, "Cylindrical antenna with tapered resistive loading," Radio Sci., vol. 2, no. 2, pp. 191-201, Feb. 1967.

[3] C. D. Taylor, "Cylindrical transmitting antenna: Tapered resistivity and multiple impedance loading," IEEE Trans. Antennas Propagat., vol. AP-16, no. 2, pp. 176-179, Mar. 1968.

[4] L. C. Shen, "An experimental study of the antenna with nonreflecting resistive loading," IEEE Trans. Antennas Propagat., vol. AP-15, no. 5, pp. 606-611, Sept. 1967.

[5] J. F. Lally and D. T. Rouch, "Experimental investigation of the broadband properties of a continuously loaded resistive monopole," IEEE Trans. Antennas Propagat., vol. AP-18, no. 6, pp. 764-768, Nov. 1970.

[6] E. E. A1tshuler, "The traveling-wave linear antenna," IEEE Trans. Antennas Propagat., vol. AP-9, no. 4, pp. 324-329, July 1961.

[7] K. Iizuka, "The traveling-wave $V$-antenna and related antennas," IEEE Trans. Antennas Propagat., v ol. AP-15, no. 2, pp. 236243, Mar. 1967.

[8] R. E. Harrington, Field Computation by Moment Methods. New York: Macmillan, 1968.

[9] C. M. Butler and D. M. Wilton, "Analysis of various numerical techniques applied to thin wire scatterers," IEEE Trans. Antennas Propagat., vol. AP-23, no. 4, pp. 534-540, July 1975.

[10] "Moment methods in electromagnetics," Supplementary Notes for a Short Course in Electromagnetic Theory, Dept. of Electrical Engineering, Univ. of Mississippi, 1975.

[11] R. R. Bowman, "Calibration techniques for electromagnetic hazard meters; $500 \mathrm{MHz}$ to $20 \mathrm{GHz}$," Nat. Bur. Stand. Internal Report 75-805, Apr. 1976.

[12] "IEEE standard definitions of terms for antennas," IEEE Std. 145-1973, Aug. 1973.

\title{
Study of Control of Beamwidth of Radiation Pattern of a Waveguide Using Inclined Slotted Flanges
}

\author{
K. K. BHAN, S. GHOSH, AND G. P. SRIVASTAVA, SENIOR MEMBER, IEEE
}

\begin{abstract}
The effect of slotted flanges on the radiation pattern of a waveguide is investigated experimentally. The slotted flanges are mounted at the aperture of the waveguide. Both straight and inclined slotted flanges are used. It is seen that considerable changes can be effected in the beamwid th of the pattern by changing the flange inclination. Such a simple control on beamwidth thus makes it possible to use the slotted flange device as a feed to meet a variety of applications.
\end{abstract}

\section{INTRODUCTION}

I T HAS BEEN shown by Srivastava et al. [1] that it is possible to develop a slotted flange system into a wide-beam low spillover feed for a deep reflector; the paper, however gives a brief summary of the results. In this paper extensive experimental evidence has been presented to establish the usefulness of the slotted flange system insofar as they are capable

Manuscript received August 30, 1976; revised March 8, 1977.

The authors are with the Department of Physics and Astrophysics, University of Delhi, Delhi-110007, India. of producing patterns suitable for efficient illumination of deep and very large reflectors having small $F / D$ ratios in the range of 0.25 to 0.5 .

\section{DESIGN CONSIDERATION}

It is well known that the radiation pattern is the Fourier transform of the aperture field distribution. A uniform aperture field distribution given by

$$
f(x)=1, \quad-1 \leqslant x \leqslant 1
$$

has a radiation pattern of the type $\sin x / x$. Conversely, a $\sin x / x$ aperture field distribution will result in a uniform (square) radiation pattern. The slotted flange, mounted symmetrically on the $E$-plane aperture of waveguide WG16, has one or more $\lambda / 4$ deep slots spaced $\lambda / 2$ apart. It is expected that alternate blocks of metal and slots will simulate a distribution of the type $\sin x / x$, and therefore produce a square radiation pattern. 